\title{
Laser-induced onset of electrospinning
}

\author{
R. Sahay, ${ }^{1}$ C. J. Teo, ${ }^{1}$ and S. T. Thoroddsen ${ }^{2}$ \\ ${ }^{1}$ Department of Mechanical Engineering, National University of Singapore, 117576 Singapore, Singapore \\ ${ }^{2}$ Division of Physical Sciences and Engineering, King Abdullah University of Science and Technology, Thuwal 23955-6900, Saudi Arabia
}

(Received 30 October 2009; published 9 March 2010)

\begin{abstract}
We present a method to start electrospinning from a polymeric drop. This method uses a pulsed laser which is focused inside the drop close to the liquid surface. The pulse cavitates the liquid and produces a protrusion from the tip of the drop. The protrusion narrows by drainage and vertical stretching, thus concentrating the electric field and increasing the charge density until it overcomes the surface tension and produces the electrified jet. This approach can reduce the required value of applied electric field to half of its value required to start convectional electrospinning from a stationary drop.
\end{abstract}

DOI: 10.1103/PhysRevE.81.035302

PACS number(s): 47.85.M-

Jetting from electrified drops has been studied since at least 1917 by Zeleny [1]. Taylor [2] carried out seminal theoretical work on the topic describing the so-called Taylor cones which form as the electric forces become sufficiently strong to overcome the surface tension. The last two decades have seen renewed interest in electrified jetting, now from polymeric drops. The resulting jets do not break up into spray, but are unstable to a bending instability, which greatly stretches the jet, leading to the production of polymeric nanofibers, in a process termed electrospinning [3,4]. As electrospinning can achieve very small diameter fibers it is ideal for fabricating mats with large surface to volume ratios, or even more regularly aligned structures [5]. The variety of potential applications ranges from filters [6], photovoltaic cells [7], photonic devices or optical sensors [8], and biomedical devices [9], such as scaffolds for bone tissue engineering [10], gas sensing [11], electron field emitters, [12] or semiconductors [13].

When high voltage is applied to a polymer drop, dissolved ions start concentrating on its surface resulting in an excess buildup of charges at the tip of the drop, which produce repulsive forces acting against the surface tension. The shape of the meniscus of the suspended polymer droplet at any particular time is thus determined by a balance of hydrostatic pressure, electrical forces and surface tension [14-18]. As the applied electric field increases, the meniscus elongates to form a conical configuration known as a Taylor cone [2]. At some critical value of the electric field, the repulsive electric forces surpass the surface tension, initiating jetting from the tip of this cone. The jet extends initially in a straight line for a certain distance (ranging from 2 to $15 \mathrm{~mm}$ ) and then suffers a catastrophic bending instability, followed by a looping and spiraling path of increasing circumference [4]. This looping and spiraling is primarily responsible for large scale elongation of the jet and is accompanied by a decreasing jet radius, further hastened by the evaporation of the solvent.

While gravity plays no role in initiating the jetting, in practice, electrospinning is sometimes started by simply turning on the liquid flow into a pendent drop and letting it grow in size until gravity detaches it from the nozzle. The pinch-off leaves a thread which stretches out between the nozzle and the drop, thereby acting as the start of the electrospinning jet. As there is no surface tension to overcome in this scenario, this spinning can start at lower than the critical voltage. However, this wastes liquid and the falling drop interferes with the formation of the nanofibers on the collection plate. Furthermore, a sessile drop sitting on a bottom platform, as in our configuration (Fig. 1), will not be pinched off by gravity. Bringing the voltage to the critical value is in some cases not practical as such high voltage very quickly drains the drop once the electrospinning starts.

Herein we employ an approach to initiate the electrospun jet at relatively lower voltages compared to the conventional process at the same operating conditions. This is done by employing a laser pulse focused just under the free surface of the drop to form a local protrusion which leads to jetting.

A different approach has recently been proposed to reduce the applied voltage in electrospinning. This method is based on striking the drop surface with a tungsten electrode so as to obtain jetting at relatively low voltages [19]. Here the wire breaks the surface neutralizing the surface tension and a thread can easily be pulled out. However, this process has the limitation of using a tungsten electrode, each time to start jetting, whereas our method is based on a noncontact approach. The presence of the eletrode is difficult to automize and interferes with the background electric field.

For larger baths of liquid, an alternative technique to promote onset of spinning, is to let bubbles rise to the surface of a liquid [20], which can produce multiple jets.

Our experimental setup consists essentially of a parallel

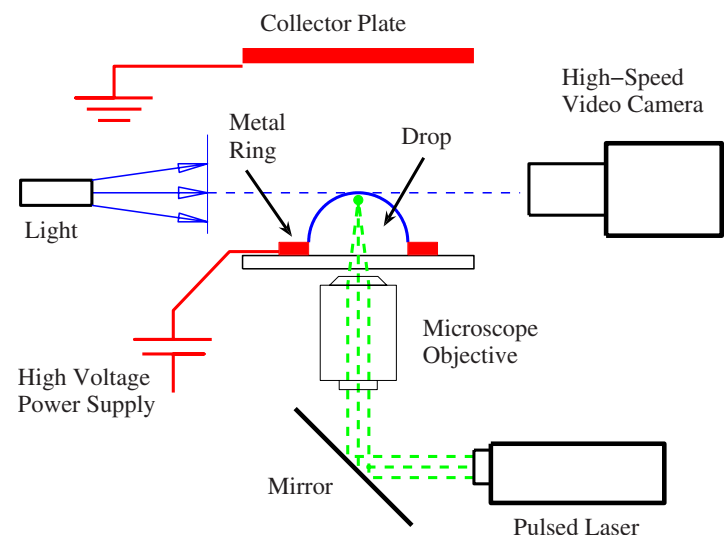

FIG. 1. (Color online) Schematic depicting the setup for laserinitiated electrospinning. Not drawn to scale. 


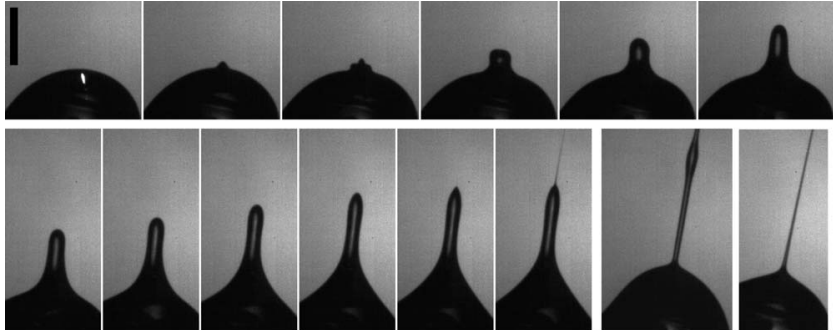

FIG. 2. The sequence of frames depicts the laser-initiation of the electrified jet, from a drop of 2 wt \% (PEO) solution. Frames are shown at $t=0.089,0.178,0.444,0.711,1.11,1.69,2.36,3.33,4.22$, $4.53,4.78,10.5$, and $400 \mathrm{~ms}$ after the laser pulse visible in the first frame. The last frame shows the jet shape much later, at $0.4 \mathrm{~s}$ after the laser pulse. The applied electric field was $3000 \mathrm{~V} / \mathrm{cm}$. Scale bar is $1.0 \mathrm{~mm}$.

plate system as shown in Fig. 1. The drop sits in a 1-mmthick metallic collar, which is $6 \mathrm{~mm}$ in outer diameter and has a central hole between $2.5-4.0 \mathrm{~mm}$ in diameter. This collar is glued to a glass plate which is about $0.8 \mathrm{~mm}$ in thickness. Hydrophobic material was sprayed on the surface of the metallic plate so as to avoid the spreading of the polymer drop, by pinning the contact line at the inner edge. The metallic collar is connected to a high voltage power supply (Glassman High Voltage, PS/MJ20P0700-22). The top circular aluminum plate, of $51 \mathrm{~mm}$ in diameter, acts as a grounding surface.

The results shown herein all used a $2 \mathrm{wt} \%$ aqueous solution of polyethylene oxide (PEO) of molecular weight $400000 \mathrm{~g} / \mathrm{mol}$ (Sigma Aldrich). We estimate the shear viscosity at $\mu \sim 50 \mathrm{cP}$ and the density and surface tension close to that of water [21].

A pulsed Nd:YAG laser (New Wave Research, model SOLO I $15 \mathrm{~Hz}$ ), with $15 \mathrm{~mJ}$ energy per pulse and duration of $5 \mathrm{~ns}$, was employed for most of the experiments. The laser beam was focused with a $20 \times$ microscopic objective directed through the drop with the beam-waist close to the top liquid surface. The location of the laser spot can be adjusted by vertically shifting the objective with a micrometer.

Most experiments used a high-speed video camera (Photron FASTCAM-Ultima APX-RS) at frame-rates up to $30 \mathrm{kfps}$, with some experiments using a Hitachi-made prototype camera [22] using frame rates up to $100 \mathrm{kfps}$. Imaging was done with a long-distance microscope (Leica Z-16 APO zoom), or Nikkor $105 \mathrm{~mm}$ macro lens, to capture the detailed close-up images of the jetting mechanism.

Figure 2 shows the laser-induced onset of electrospinning. Here the drop is in a stationary electric field when a laser pulse is applied, at $\sim 200 \mu \mathrm{m}$ below the surface. The strength of the electric field is here $3000 \mathrm{~V} / \mathrm{cm}$, which is less than half of the critical value, $E_{c} \simeq 6900 \mathrm{~V} / \mathrm{cm}$, which is needed to produce a singular Taylor cone in our configuration. This critical value was determined by slowly increasing the voltage in experiments without the laser pulse.

The laser cavitates the liquid, forming an expanding plasma under the surface, which forces out a protrusion from the top of the drop. The width of this protrusion is initially about $350 \mu \mathrm{m}$ (third frame) and it emerges at $1 \mathrm{~m} / \mathrm{s}$. The top

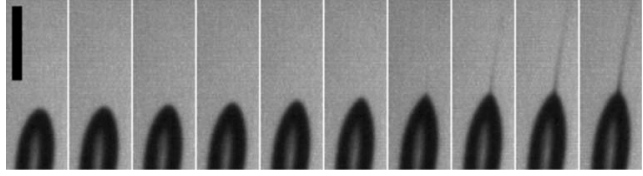

(a)

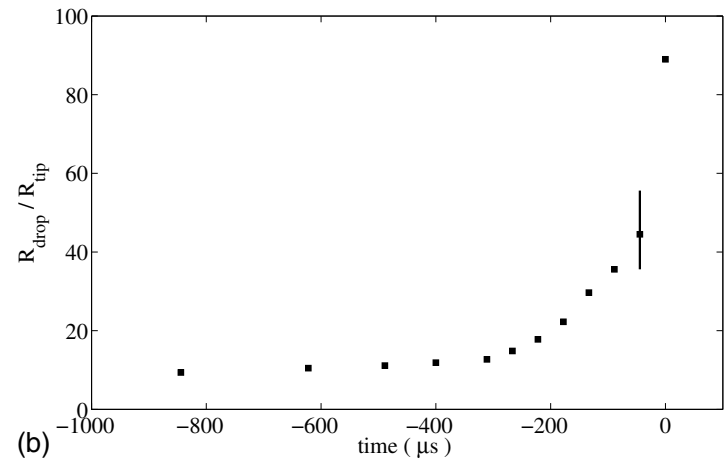

FIG. 3. (a) Close-up images of the onset of the microjet, from the same sequence as in Fig. 2. The frames are $44.4 \mu$ s apart. Scale bar is $0.5 \mathrm{~mm}$. (b) The increase in tip curvature vs time approaching the microjetting. The tip-curvature is normalized by the undisturbed drop curvature.

of the protrusion decelerates to $0.2 \mathrm{~m} / \mathrm{s}$ in the second row of Fig. 2 where the overall electric field pulling it up and the capillary force pulling the top down are approaching a balance. At the same time liquid is being drained back into the drop by the excess capillary pressure $p_{\sigma} \simeq \sigma / R_{p}$, where $R_{p}$ represents the azimuthal radius of the protrusion. The protrusion therefore becomes thinner both by drainage and stretching. The presence of this protrusion greatly concentrates the electric field. Furthermore, the reduction in surface area concentrates the electric charge and the surface curvature at the tip increases until it becomes unstable, sending out the microjet $4.5 \mathrm{~ms}$ after the laser pulse. Figure 3(a) shows close-up images of the tip of the protrusion when the electrified fine jet emerges at much higher velocity $(\simeq 11 \mathrm{~m} / \mathrm{s})$. In Fig. 3(b)
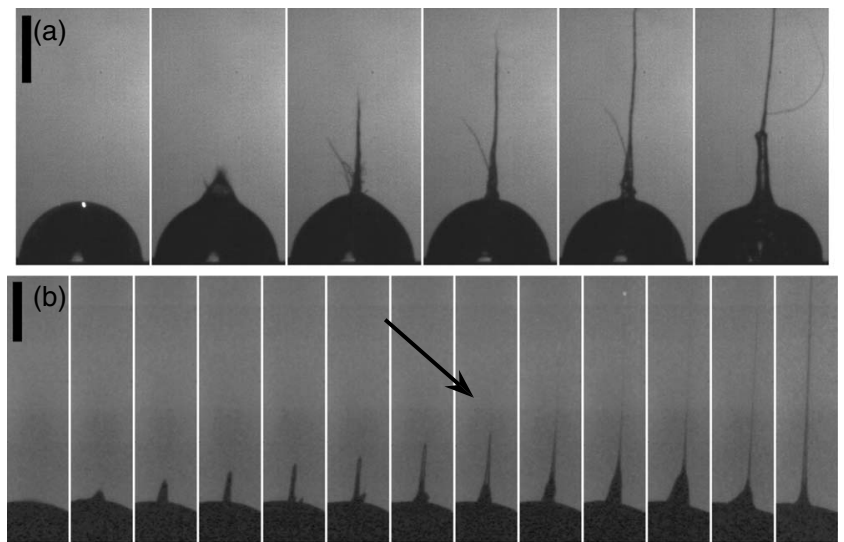

FIG. 4. (a) Jetting for a laser spot very close to the surface of the drop $(<100 \mu \mathrm{m})$. The first 5 frames are spaced by $44.4 \mu$ s with the last frame $667 \mu$ s after the laser pulse. The applied electric field was $3000 \mathrm{~V} / \mathrm{cm}$. (b) Jetting from a $10 \mathrm{~mJ}$ laser pulse. First 8 frames are spaced by $d t=20 \mu \mathrm{s}$, with the last one $2.4 \mathrm{~ms}$ later. Scale bars are $1 \mathrm{~mm}$. 
we estimate the surface curvature at the top of the protrusion, leading up to the ejection time. When the jet emerges, the tip curvature is $\sim 60$ times higher than the drop curvature. The half-cone angle appears close to the critical angle observed in the literature, i.e., around $49^{\circ}[2,15]$.

The most important control parameter in our mechanism is the depth of the point-of-focus of the laser pulse, below the drop surface. If the laser is focused too close an ejecta crown emerges out of the surface, producing irregular splashing [23-25]. Figure 4(a) shows a laser close to the free surface, which opens a crown resulting in irregular side-jets alongside the main vertical jet. Regular concentrated protrusions are generated when the laser depth is between $\sim 150-500 \mu \mathrm{m}$, for the $15 \mathrm{~mJ}$ pulse energy. Higher pulseenergy will change this range. Figure 4(b) shows regular jetting for a weaker $10 \mathrm{~mJ}$ pulse. Here the protrusion is pointier and is missing the thicker bead prominent in Fig. 2. The microjet emerges after $140 \mu \mathrm{s}$.

These results are robust but can clearly be optimized by adjusting the laser depth and pulse energy. Gravity plays no role in the present technique and the drop could as easily be pendent from a short nozzle. Additionally, onset of jetting must be followed by the appropriate field strength and flow rate into the drop. Our high-speed videos show sustained regular spinning persisting over at least $0.5 \mathrm{~s}$, following the laser pulse. However, our setup does not allow for inflow into the drop to replenish it and we cannot tell if the spinning has reached a fully steady state.

One drawback of the technique is that it will not work with opague liquids. In those cases, laser ablation might work, where the laser is directed through the air onto the liquid surface.

One can easily envision a fully automated system based on our method. It would simply turn on the voltage $V_{o}$ and flow-rate $Q_{o}$ into the drop at the desired value for continuous spinning. The size of the drop would then grow until it cuts a continuous laser/photodiode trigger, which initiates the laser-pulse thus starting the spinning at the desired $V_{o}$ and $Q_{o}$.

We thank T. G. Etoh, K. Takehara, and H. Nakano at Kinki University for their help with the high-speed imaging in Fig. 4(b).
[1] J. Zeleny, Phys. Rev. 10, 1 (1917).

[2] G. I. Taylor, Proc. R. Soc. London, Ser. A 313, 453 (1969).

[3] D. H. Reneker and I. Chun, Nanotechnology 7, 216 (1996).

[4] D. H. Reneker et al., J. Appl. Phys. 87, 4531 (2000).

[5] A. Theron, E. Zussman, and A. L. Yarin, Nanotechnology 12, 384 (2001)

[6] H. J. Jin, S. V. Fridrikh, G. C. Rutledge, and D. L. Kaplan, Biomacromolecules 3, 1233 (2002).

[7] H. S. Shim, S. I. Na, S. H. Nam, H. J. Ahn, H. J. Kim, D. Y. Kim, and W. B. Kim, Appl. Phys. Lett. 92, 183107 (2008).

[8] X. Y. Wang, C. Drew, S.-H. Lee, K. J. Senecal, J. Kumar, and L. A. Samuelson, Nano Lett. 2, 1273 (2002).

[9] H. L. Schreuder-Gibson and P. Gibson, ACS Symp. Ser. 918, 121 (2006).

[10] H. Yoshimoto, Y. M. Shin, H. Terai, and J. P. Vacanti, Biomaterials 24, 2077 (2003).

[11] S. Piperno, M. Passacantando, S. Santucci, L. Lozzi, and S. La Rosa, J. Appl. Phys. 101, 124504 (2007).

[12] X. C. Ma, E. G. Wang, W. Z. Zhou, D. A. Jefferson, J. Chen, S. Z. Deng, N. S. Xu, and J. Yuan, Appl. Phys. Lett. 75, 3105 (1999).

[13] J. Yu, J. Ahn, S. F. Yoon, Q. Zhang, Rusli, B. Gan, K. Chew, M. B. Yu, X. D. Bai, and E. G. Wang, Appl. Phys. Lett. 77, 1949 (2000).
[14] A. F. Spivak and Y. A. Dzenis, Electrostatics 1999 (IOP Publishing Ltd, Bristol, 1999), Vol. 163, pp. 175-178.

[15] A. L. Yarin, S. Koombhongse, and D. H. Reneker, J. Appl. Phys. 90, 4836 (2001).

[16] M. M. Hohman, M. Shin, G. Rutledge, and M. P. Brenner, Phys. Fluids 13, 2201 (2001).

[17] J. J. Feng, Phys. Fluids 14, 3912 (2002).

[18] Y. M. Shin, M. M. Hohman, M. P. Brenner, and G. C. Rutledge, Polymer 42, 09955 (2001).

[19] C. Chang, K. Limkrailassiri and L. W. Lin, Appl. Phys. Lett. 93, 123111 (2008).

[20] Y. Liu and J. H. He, Int. J. Nonlinear Sci. Numer. Simul. 8, 393 (2007).

[21] H. Fong, I. Chun, and D. H. Reneker, Polymer 40, 4585 (1999).

[22] H. Ohtake, T. Hayashida, K. Kitamura, T. Arai, J. Yonai, K. Tanioka, H. Maruyama, and T. G. Etoh, Broadcast Technol. 28, 2 (2006).

[23] D. Obreschkow, P. Kobel, N. Dorsaz, A. de Bosset, C. Nicollier, and M. Farhat, Phys. Rev. Lett. 97, 094502 (2006).

[24] I. Apitz and A. Vogel, Appl. Phys. A: Mater. Sci. Process. 81, 329 (2005).

[25] S. T. Thoroddsen, K. Takehara, T. G. Etoh, and C.-D. Ohl, Phys. Fluids 21, 112101 (2009). 